\title{
Improving Link Quality of UWB Communication Links by Means of PSWF-Basis Persuit Denoising
}

\author{
Wouter Dullaert ${ }^{1}$ Hendrik Rogier ${ }^{2}$ Luca De Camillis ${ }^{3}$ Tom Dhaene ${ }^{4}$
}

\begin{abstract}
Wireless Body Area Networks (WBANs) utilising Ultra Wideband communication channels often use a combination of thin sensor nodes and a heavy base node. The sensor nodes need to be as simple and low power as possible. This makes it difficult to maintain a good quality link. In this paper additional post processing steps at the base node are proposed in order to overcome these issues. A basis persuit denoising technique, using Prolate Spheroidal Wave Functions (PSWFs), combined with a sampling technique inspired by compressive sensing allows data to be recovered even when the link is interrupted for short intervals.
\end{abstract}

\section{INTRODUCTION}

Ultra Wideband (UWB) communications are a natural fit for Wireless Body Area Networks. The combination of unlicensed use with a low spectral density, which allows co-existence with other communication systems, opens up a lot of possibilities. WBANs often consist of simple low-power sensor nodes that transmit their data to a central, more powerful node. Cost and power limitations make the communication link very weak and put restrictions on the amount of processing that can be performed at the sensor nodes. Additional processing steps to improve the quality of the communication link should therefore be located at the central node.

In this paper basis persuit denoising is proposed to improve the link quality. Basis persuit denoising assumes that the relevant signal is sparse in a given basis and that the added noise is not. By finding this sparse representation of the signal an accurate representation of the original signal can be obtained. Of course the basis used has a great impact on the performance of this technique. In this paper Prolate Spheroidal Wave Functions (PSWFs) will be used. In order to obtain these coefficients an L1 optimisation algorithm will be used.

This paper is organised as follows: in Section 2 the PSWF-basis is introduced. The L1 optimisation to obtain the coefficients of a signal in this basis is presented in Section 3. A proof of concept is provided in Section 4. Finally, the paper is wrapped up with the conclusions in Section 5.

\section{Prolate Spheroidal Wave function Ba- SIS}

The Prolate Spheroidal Wave Function arises when solving the scalar wave equation in prolate spheroidal coordinates. In 1961 Slepian et al. proved, [1], that they are also the solution to the following maximisation problem:

$$
\alpha(T)^{2}=\frac{\int_{-T}^{T}|f(t)|^{2}}{\int_{-\infty}^{\infty}|f(t)|^{2}}
$$

where $f(t)$ is $2 B$-bandlimited. This means that the zero-th order PSWF $\psi_{c, 0}(t)$ maximises $\alpha(T)^{2}$ in (1). When this excercise is repeated over the space containing all functions orthogonal to $\psi_{c, 0}(t)$, the first-order PSWF $\psi_{c, 1}(t)$ will maximise (1), etc. As can be seen from the notation, the PSWFs are also function of the time-bandwidth product $c=2 B T$. In this paper $c$ can be freely chosen to best suit the problem at hand. In order not to overload the notation we will suppress the dependency on $c$ where it is not of particular interest.

The PSWFs are imbued with a lot of nice properties. Of particular interest is the fact that they are orthogonal over both $[-\infty, \infty]$ and $[-T, T]$. They are also complete in $[-T, T]$ and complete for all bandlimited functions over the entire time interval $[-\infty, \infty]$. They can also be scaled to have unit norm in either region. These properties allow any bandlimited signal $s(f)$ to be represented as a series of PSWFs:

$$
s(f)=\sum_{k=0}^{\infty} A_{k} \psi_{k}(f)
$$

In [1], Slepian also showed that the energy of a PSWFs $\psi_{k}(f)$ present in $[-T, T]$ decreases rapidly

\footnotetext{
${ }^{1}$ Department of Information Technology, Ghent University, Sint-Pietersnieuwstraat 41 B-9000 Ghent, email: wouter.dullaert@intec.ugent.be

${ }^{2}$ Department of Information Technology, Ghent University, Sint-Pietersnieuwstraat 41 B-9000 Ghent, email: hendrik.rogier@intec.ugent.be

${ }^{3}$ Department of Information Technology, Ghent University, Sint-Pietersnieuwstraat 41 B-9000 Ghent, email: luca.de.camillis@intec.ugent.be

${ }^{4}$ Department of Information Technology, Ghent University, Sint-Pietersnieuwstraat 41 B-9000 Ghent, email: tom.dhaene@ugent.be
} 
when its order $k$ increases. This means that most physical signals, which have both limited bandwidth and duration, are sparse in a PSWF-basis when using an appropriate time-bandwidth constant $c$. In [2], [3] this is explained in more detail for the discrete prolate spheroidal sequence, the discrete counterpart of the PSWF.

\section{L1 Optimisation}

The purpose of the basis-persuit denoising technique is to find the coefficients $A_{k}$ from (2) which best represent the unknown original function $s(f)$. A straightforward approach would be to project the received signal $r(f)=s(f)+n(f)$, where $n(f)$ is a noise contribution, onto the PSWF basis using:

$$
A_{k}=\int_{-B / 2}^{B / 2} r(f) \psi_{k}(f) d f
$$

In practice (3) can only be accurately evaluated using a Gauss-Legendre quadrature, which puts great restrictions on its application. Either $r(f)$ must be sampled directly on a Guass-Legendre quadrature or it must be interpolated onto such a quadrature, both of which have their own set of difficulties.

This problem can be avoided by not using (3) to calculate the coefficients $A_{k}$, but by iteratively searching for the coefficients using a proper optimisation algorithm. This technique also forms the basis of socalled compressive sensing schemes, first presented in [4]. The reasoning is that because $s(f)$ is sparse, or nearly sparse, when expanded into PSWFs, of all sets $\left\{A_{k}\right\}$ that are consistent with $r(f)$ the one with the least amount of coefficients will provide the best approximation of $s(f)$. This is equivalent to the constrained minimisation of the L0-norm of the $A_{k}$, subject to limiting the mean-square error (MSE) between $\hat{s}(f)$, the estimation $s(f)$, and $r(f)$

$$
\min _{A_{k}}\left\|A_{k}\right\|_{0} \quad \text { subject to } \quad|r(f)-\hat{s}(f)|^{2}<\epsilon
$$

where the L0-norm, defined as $\left\|A_{k}\right\|_{0}=\sum_{k=0}^{K} A_{k}^{0}$ is the amount of nonzero $A_{k}$, for some suitable value of $\epsilon$. Unfortunately this problem is NP complete. However, [4] also shows that in most cases minimising the L1norm leads to equivalent results as mimimising the L0norm:

$$
\min _{A_{k}}\left\|A_{k}\right\|_{1} \quad \text { subject to } \quad|r(f)-\hat{s}(f)|^{2}<\epsilon
$$

with $\left\|A_{k}\right\|_{1}=\sum_{k=0}^{K}\left|A_{k}\right|$. Various algorithms to efficiently mimimise (5) exist. Another important result from [4] states, that if the sensing matrix satisfies the
Restricted Isometry Property, ie. if its columns are almost orthogonal, and using a measurement vector of length $m \geq C S \log N / S$ samples:

- the recovery $s(f)$ is exact when $s(f)$ is sparse

- the MSE between $s(f)$ and its reconstruction $\hat{s}(f)$ is optimal using only $S$ coefficients when $s(f)$ is compressible, but not exactly sparse.

where $N$ is the length of $\hat{s}(f), S$ the maximum amount of basis functions and $C$ a small constant that is problem dependent. As a rule of thumb, between 2 and 4 samples should be taken for each unknown coefficient $A_{k}$, to allow for optimal reconstruction. The advantage of this approach is that it does not impose any restrictions on the sampling. In fact the more randomly distributed the samples are, the higher the probability becomes that the optimisation from (5) converges to the right solution. The downside is that this is computationally more difficult to achieve than (3).

\section{Validation}

We will validate this technique on both the magnitude $\left|H_{t x}(f)\right|$ and phase $\angle H_{t x}(f)$ of the transfer function of the bowtie antenna from [5]. The transfer function was measured between $400 \mathrm{MHz}$ and $20 \mathrm{GHz}$ using 801 samples. From these samples a subset is randomly chosen, which serves as the measurement vector $y$. (5) is then solved using the CVX and SeDuMi matlab toolboxes. For the magnitude of the transfer function $\left|H_{t x}(f)\right|, 50$ PSWF coefficients $A_{k}$ will be estimated, for the phase $\angle H_{t x}(f), 30$ coeffients. Using the rule of thumb, this means we need about 100 measurements for $\left|H_{t x}(f)\right|$ and 60 measurements for $\angle H_{t x}(f)$. Figure 1 and 2 shows the original funtion, its estimate after L1 minimisation and the measurement vector $y$ for $\left|H_{t x}(f)\right|$ and $\angle H_{t x}(f)$ respectively. It can be seen that the reconstruction of $\angle H_{t x}(f)$ is better than that of $\left|H_{t x}(f)\right|$, but that in both cases the result is quite good.

Figures 3 and 4 show the estimated coefficients $\left|H_{t x}(f)\right|$ and $\angle H_{t x}(f)$ respectively. We can now see why the algorithm performed better on $\angle H_{t x}(f)$ : this function approximates the initial assumption of sparsity better than $\left|H_{t x}(f)\right|$. Its coefficients go smoothly to zero, while the coefficients of $\left|H_{t x}(f)\right|$ never quite settle down to 0 when the order $k$ increases, due to a combination of measurement noise and small signal contributions.

On Figures 5 and 6 the relative error between the original function and its reconstruction, defined as:

$$
\text { Error }=\frac{|s(f)-\hat{s}(f)|}{s(f)}
$$

with $s(f)$ the original and $\hat{s}(f)$ the reconstructed function, is shown for $\left|H_{t x}(f)\right|$ and $\angle H_{t x}(f)$ respectively. 


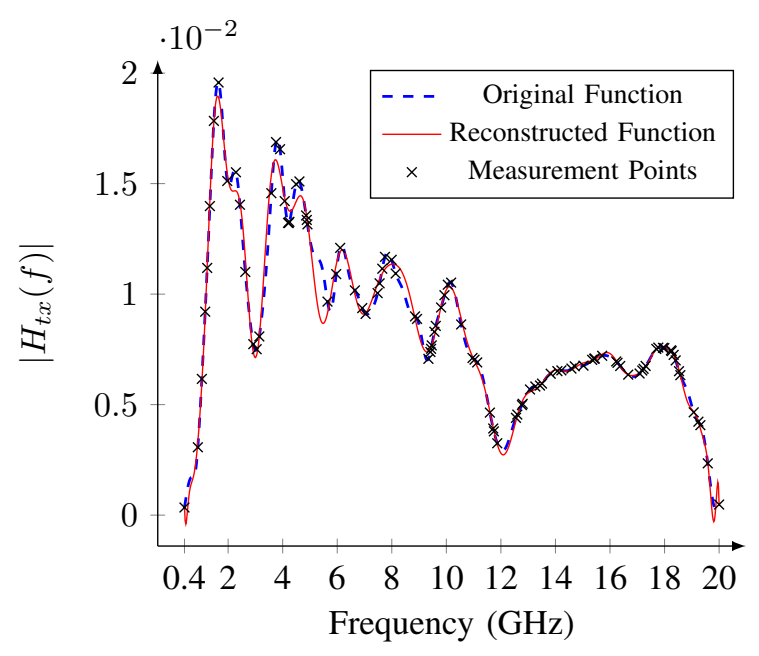

Figure 1. Original and reconstructed $\left|H_{t x}(f)\right|$

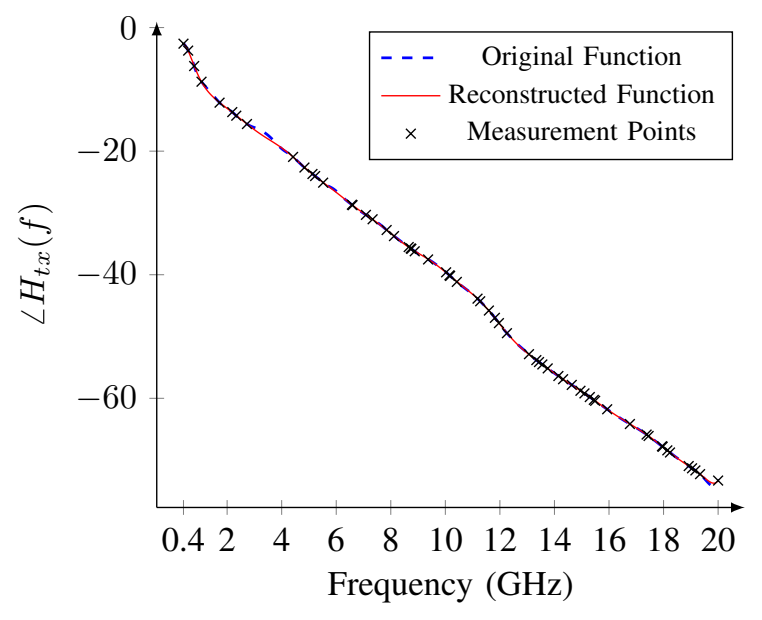

Figure 2. Original and reconstructed phase of $H_{t x}(f)$

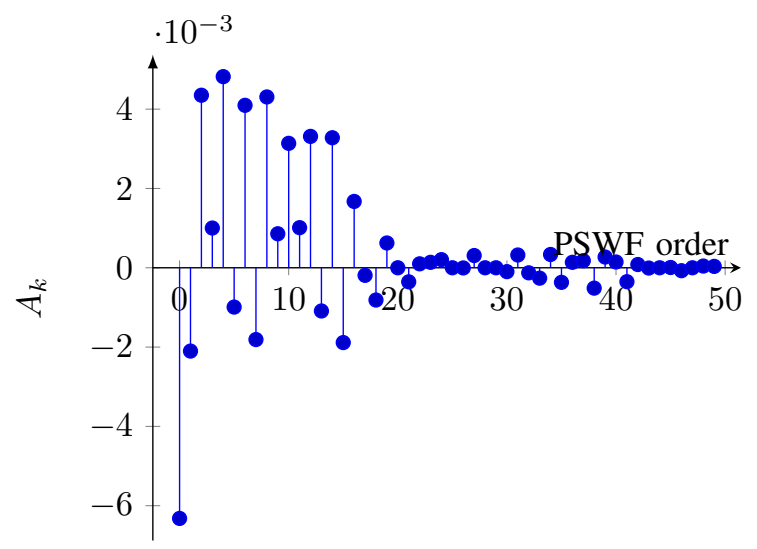

Figure 3. Estimated PSWF coefficients of $\left|H_{t x}(f)\right|$

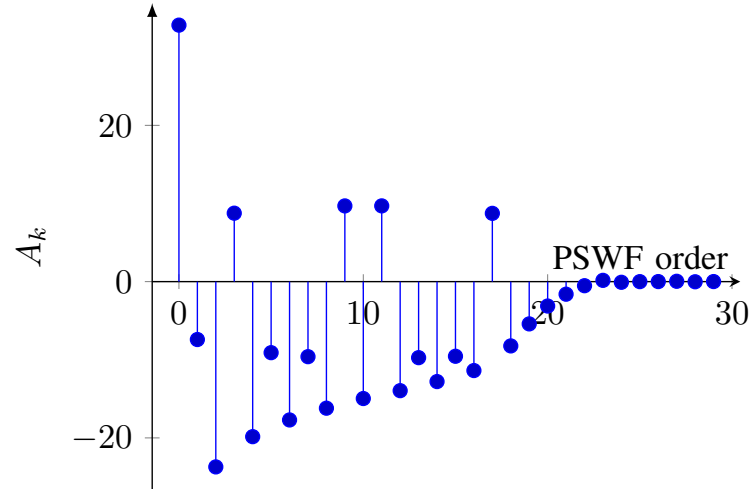

Figure 4. Estimated PSWF coefficients of the phase of $H_{t x}(f)$

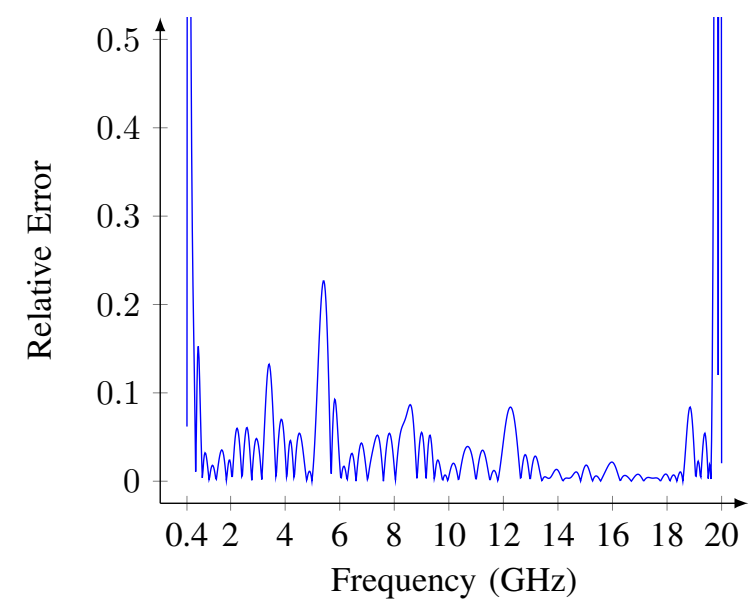

Figure 5. Relative error on $\left|H_{t x}(f)\right|$

The relative error comfirms the previous results: the reconstruction is very good for $\angle H_{t x}(f)$ with a relative error below $5 \%$. The relative error on $\left|H_{t x}(f)\right|$ is worse: very high spikes at the sides and a few spikes above $10 \%$ otherwise. The high error level at the sides is largely due to the fact that $\left|H_{t x}(f)\right|$ is very low at these frequencies, which increases the relative error disproportionally. The other spikes are due to the fact that $\left|H_{t x}(f)\right|$ is not perfectly sparse and hence not all information can be reconstructed using just 50 coefficients $A_{k}$.

\section{Conclusion}

A new method to acquire UWB signals, based on the compressive sensing framework has been presented. It allows the reconstruction of the transmitted signal using a very limited amount of arbitrarily located samples, greatly reducing the requirements on the hardware and channel at the cost of additional post processing. The technique works by assuming that the transmitted signal is sparse in a basis of prolate spheroidal wave functions. This assumption is broadly satisfied for most 


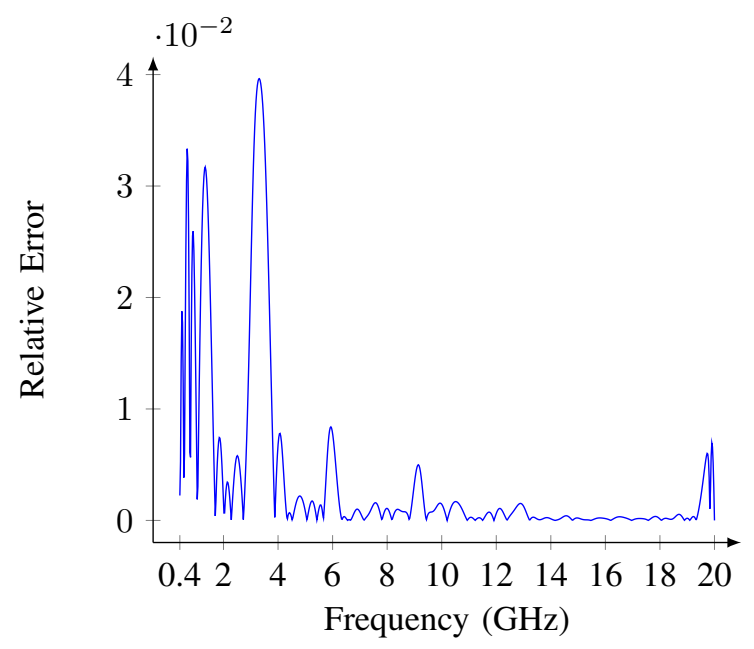

Figure 6. Relative error on the phase of $H_{t x}(f)$

physical systems. The accuracy of the reconstruction is directly related to how well the assumption of sparsity holds. Unfortunately the performance degrades rapidly if the signal is not completely sparse. Improvements to the robustness is a topic for further research.

\section{References}

[1] D. Slepian and H.O. Pollak, "Prolate Spheroidal Wave Functions, Fourier Analysis, and Uncertainty - I," Bell Systems Technology Journal, vol. 40, no. 1, pp. 43-64, Jan. 1961.

[2] W. Dullaert and H. Rogier, "Novel Compact Model for the Radiation Pattern of UWB Antennas Using Vector Spherical and Slepian Decomposition," IEEE Transactions on Antennas and Propagation, vol. 58, no. 2, pp. 287-299, 2010. [Online]. Available: http://ieeexplore.ieee.org/lpdocs/epic03/wrapper.htm? arnumber $=5345757$

[3] W. Dullaert, G. Adamiuk, and H. Rogier, "Compression of measured 2D UWB antenna transfer functions," Electronics Letters, vol. 46, no. 8, p. 552, 2010. [Online]. Available: http: //ieeexplore.ieee.org/xpl/freeabs $\backslash$ all.jsp?arnumber $=5451000$

[4] E. Candes, J. Romberg, and T. Tao, "Robust uncertainty principles: exact signal reconstruction from highly incomplete frequency information," IEEE Transactions on Information Theory, vol. 52, no. 2, pp. 489-509, Feb. 2006. [Online] Available: http://ieeexplore.ieee.org/lpdocs/epic03/wrapper.htm? arnumber $=1580791$

[5] W. Wiesbeck, G. Adamiuk, and C. Sturm, "Basic Properties and Design Principles of UWB Antennas," Proceedings of the IEEE, vol. 97, no. 2, pp. 372-385, 2009. [Online]. Available: http://ieeexplore.ieee.org/lpdocs/epic03/wrapper.htm? arnumber $=4802200$ 